\title{
Mutation in the Tight-Junction Gene Claudin 19 (CLDN19) and Familial Hypomagnesemia, Hypercalciuria, Nephrocalcinosis (FHHNC) and Severe Ocular Disease
}

\author{
Muhammad Naeem $^{a}$ Sofia Hussain ${ }^{a}$ Naureen Akhtar ${ }^{b}$ \\ ${ }^{a}$ Department of Biotechnology, Faculty of Biological Sciences, Quaid-i-Azam University, Islamabad, and \\ ${ }^{b}$ Department of Nephrology, The Children's Hospital and The Institute of Child Health, Lahore, Pakistan
}

\section{Key Words}

Hypomagnesemia • Ocular involvement • Claudin $19 \cdot$

Nephrocalcinosis $\cdot$ Tight junction

\begin{abstract}
Background/Aims: Familial hypomagnesemia with hypercalciuria and nephrocalcinosis (FHHNC) is a rare renal tubular disorder complicated by progressive renal failure during childhood or adolescence. Recently, causative mutations in the CLDN19 gene have been identified in FHHNC patients presenting with severe ocular involvement. The aim of the study was to investigate the molecular genetic defect underlying FHHNC in a consanguineous Pakistani family. Methods: Clinical and biochemical parameters of the proband were studied during the follow-up period over 5 years. Genotyping of 7 members of the family was performed by amplifying microsatellite markers, tightly linked to the CLDN16 and CLDN19 genes. The two genes were sequenced directly in an automated sequencer. PCR-RFLP assay and bioinformatic analysis were performed to verify the identified mutation. Results: Genotyping revealed that the proband was homozygous for the marker loci tightly linked to the CLDN19 gene. Sequence analysis in the proband revealed homozy-
\end{abstract}

gosity for a novel missense mutation in exon 3 of the CLDN19 gene (389G $>A$ ) resulting in G130D amino acid substitution. Bioinformatic analysis supported the pathogenicity of the identified mutation. Family screening revealed nephrolithiasis in 3 of $6(50 \%)$ heterozygous carriers of the pathogenic mutation. Conclusion: This study supports the fundamental role of claudin 19 for magnesium homeostasis, normal tubular structures in the kidney, and undisturbed organization and development of the retina.

Copyright $\odot 2011$ S. Karger AG, Basel

\section{Introduction}

Familial hypomagnesemia with hypercalciuria and nephrocalcinosis (FHHNC) was first described in 1972 [1] and is characterized by excessive renal magnesium and calcium wasting and nephrocalcinosis [2]. The disorder is inherited in an autosomal recessive fashion and usually leads to end-stage renal disease (ESRD) in the second to fourth decades of life. The clinical appearance in affected patients is often uniform, although time to ESRD shows considerable variation, even among siblings. All affected individuals exhibit marked hypomagnesemia

\section{KARGER}

Fax +41613061234 E-Mail karger@karger.ch www.karger.com

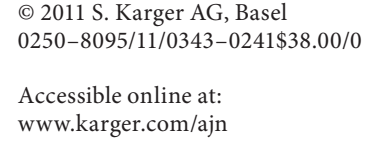

Assist. Prof., Dr. Muhammad Naeem

Department of Biotechnology

Faculty of Biological Sciences, Quaid-i-Azam University

Islamabad 45320 (Pakistan)

Tel. +92 519064 4119, E-Mail mnaeem@ qau.edu.pk 
with hypercalciuria and nephrocalcinosis. The severity of nephrocalcinosis is usually correlated with rapid progression to ESRD, but this association was disputed in some of the literature [3-6]. Other symptoms at onset include recurrent urinary tract infections, nephrolithiasis, polyuria, polydipsia, failure to thrive, muscular tetany, hypocitraturia, impaired glomerular filtration rate and incomplete distal renal tubular acidosis [7]. Ocular abnormalities and hearing impairment have been reported as inconsistent extrarenal findings. The only known cure is renal transplantation; however, symptomatic treatment in the form of magnesium citrate and calcium replacement, or the use of hydrochlorothiazide only delays the onset of ESRD [3]. Mutations in the gene CLDN16 mapping to chromosome $3 \mathrm{q} 27$ were identified as the underlying genetic defects in FHHNC. CLDN16 encodes claudin 16 (CLDN16), a tight-junction protein of the claudin multigene family that is expressed in the thick ascending limb of Henle (TALH) and the distal convoluted tubule [8]. Several mutations in the CLDN16 have been reported so far.

Konrad et al. [9] mapped another gene (CLDN19) for autosomal recessive renal magnesium loss to chromosome 1p34.2 in patients affected by FHHNC and significant ocular abnormalities, including macular colobomata, nystagmus and myopia. CLDN19 encodes for claudin 19 (CLDN19), a recently identified member of the claudin multigene family. CLDN19 is expressed at high levels in the kidney and the eye. To date, six different mutations have been reported in the CLDN19 gene $[9,10]$. In this paper, we describe a novel CLDN19 mutation in a Pakistani family affected with FHHNC and severe ocular disease.

\section{Subjects and Methods}

The proband was a 19-year-old boy from a consanguineous Pakistani family (fig. 1a) living in a remote village in Punjab province. The proband and his family members were clinically examined at the Children's Hospital and the Institute of Child Health, Lahore, Pakistan. Before the start of the study, approval was obtained from the Quaid-i-Azam University institutional review board. In addition, informed consent was obtained from the family members (III-1, III-2, IV-1, IV-4, IV-6, IV-7 and IV-8) who participated in the study.

Venous blood samples were obtained from 7 family members and genomic DNA was extracted from whole blood following a standard protocol. Microsatellite markers tightly linked to the CLDN16 (D3S3686, D3S3651, D3S3628) and CLDN19 (D1S3721, D1S2130, D1S2645) genes were polymerase chain reaction (PCR) amplified from genomic DNA. The PCR was carried out accord- ing to a standard procedure in $50-\mu l$ reaction volumes containing $100 \mathrm{ng}$ of genomic DNA, $20 \mathrm{pmol}$ of each primer, $200 \mu \mathrm{M}$ of each deoxynucleoside triphosphate, 2.5 units of Taq DNA polymerase (MBI Fermentas) and $1 \times$ PCR buffer. The thermal cycling conditions used were: $95^{\circ} \mathrm{C}$ for $5 \mathrm{~min}$, followed by 40 cycles of $95^{\circ} \mathrm{C}$ for $1 \mathrm{~min}, 53-58^{\circ} \mathrm{C}$ for $1 \mathrm{~min}, 72^{\circ} \mathrm{C}$ for $1 \mathrm{~min}$, and a final extension at $72^{\circ} \mathrm{C}$ for $10 \mathrm{~min}$. PCR was performed by use of the Palm-Cycler gradient thermal cycler (Corbett Life Science, Australia). The PCR products were resolved on $8 \%$ non-denaturing polyacrylamide gel and genotypes were assigned by visual inspection.

To screen for the mutation, each of the exons and adjacent exon/intron boundaries of the CLDN16 and CLDN19 genes were PCR amplified from genomic DNA using primers designed from intronic sequences of the two genes. The sequences of the primers are available upon request. PCR products were purified using the Gene JET PCR purification kit (Fermentas Life Sciences) and were sequenced in Applied Biosystems automatic sequencer 3730XL, using the BigDye terminator cycle sequencing kit V3.1 (PE Applied Biosystems, Foster City, Calif., USA) following purification by ethanol precipitation. Sequence variants were identified using BioEdit Sequence Alignment Editor version 7.0.5.3.

The identified mutation obliterated the restriction site for Stul enzyme, so its presence was assayed by PCR amplification of CLDN19 exon 3 from genomic DNA, digestion of the product with Stul (Fast Digest, Fermentas Life Sciences), and separation of the resulting fragments by agarose gel electrophoresis with direct visualization using ethidium bromide.

Fig. 1. a The family pedigree of the individual affected with FHHNC with haplotypes for the most closely linked markers shown below each symbol. b-d Radiological examination showing renal osteodystrophy and genu valgum (knock-knee) deformity at the age of 19 years. e-g Fundoscopic images showing macular colobomata in the left eye, posterior staphyloma in the right eye, increased tortuosity of the retinal arteriolar vessels in both eyes $(\mathbf{e}, \mathbf{f})$, and left exotropia $(\mathbf{g})$. $\mathbf{h}$ DNA sequence analysis of the gene from a homozygous affected individual (top panel), a heterozygous carrier (middle panel), and a control individual (bottom panel). The arrow denotes nucleotide position 389 , which is mutated from $\mathrm{G}$ to A, resulting in a G130D missense mutation. i An ethidium bromide-stained agarose gel containing a 574-bp amplified product that was digested with Stu1. The digested PCR products from a normal control individual, heterozygous carriers (III1, III-2, IV-1, IV-4, IV-6 and IV-7) of the family and an affected individual (IV-8) are shown. The normal PCR product was digested into 385- and 189-bp fragments, whereas the 574-bp products with the G130D defect were not digested. A 100-bp DNA ladder is shown on the left. $\mathbf{j}$ Amino acid sequence alignment of the third transmembrane domain of claudin 19 in 18 vertebrate species. The G130 residue (indicated by an arrow) was completely conserved among these species. $\mathbf{k}$ Schematic representation of the claudin-19 protein and G130D mutation. TM = Transmembrane domain; $\mathrm{C}=$ carboxy terminal; $\mathrm{N}=$ amino terminal. 


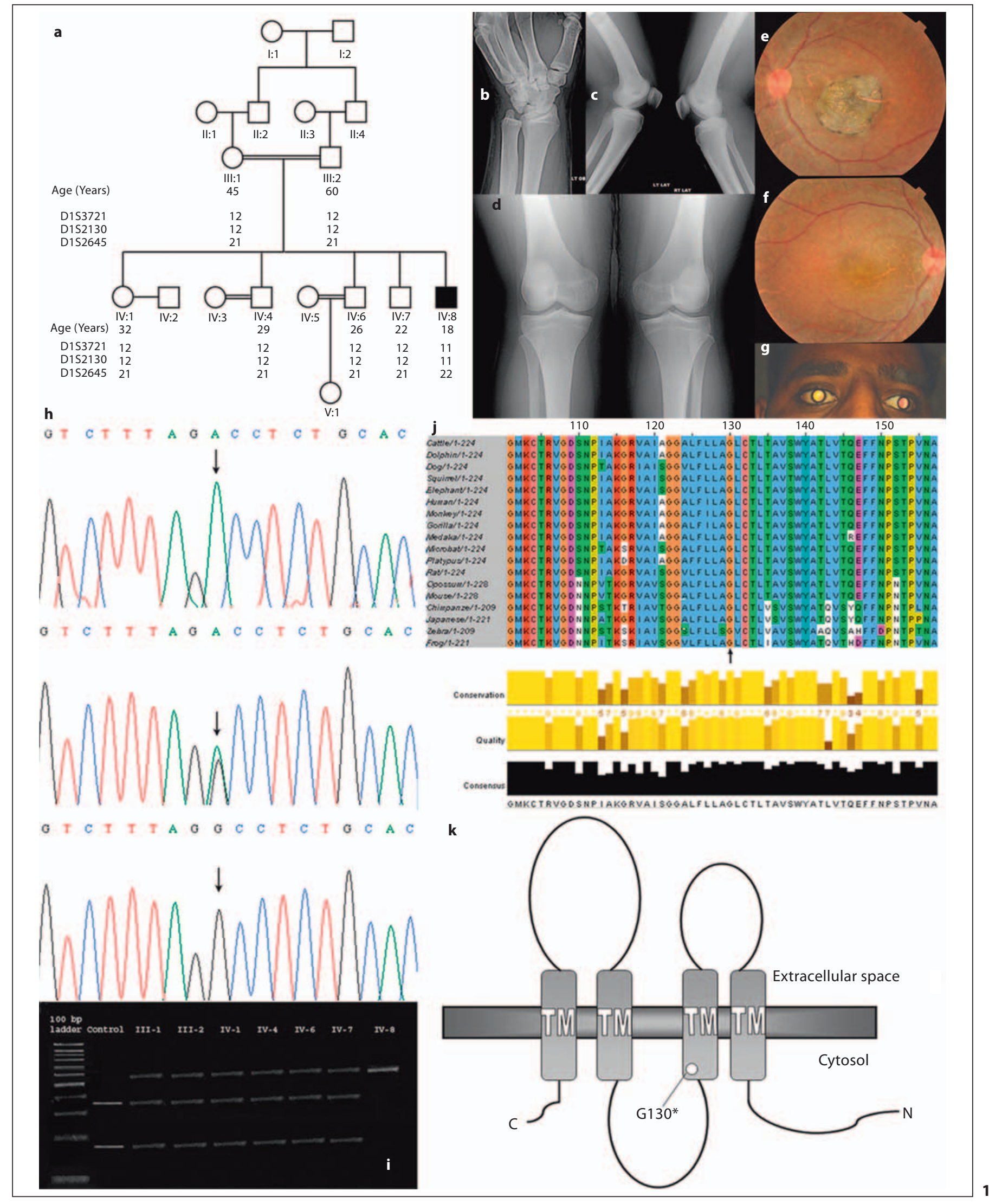

A Pakistani FHHNC Family with

Am J Nephrol 2011;34:241-248 
Table 1. Biochemical characteristics of the patient recorded during the follow-up period

\begin{tabular}{|c|c|c|c|c|c|}
\hline \multirow[t]{2}{*}{ Parameters } & \multirow{2}{*}{$\begin{array}{l}\text { Reference } \\
\text { ranges }\end{array}$} & \multicolumn{4}{|c|}{ Age, years } \\
\hline & & 14 & 15 & 16 & 19 \\
\hline Serum $\mathrm{Ca}^{2+}, \mathrm{mg} / \mathrm{dl}$ & $8.8-10.2$ & 6.1 & 6.8 & 7.9 & 7.6 \\
\hline Serum $\mathrm{Mg}^{2+}, \mathrm{mg} / \mathrm{dl}$ & $1.6-2.5$ & 0.5 & 1.4 & 1.5 & 1.5 \\
\hline Serum creatinine, $\mathrm{mg} / \mathrm{dl}$ & $0.4-1.3$ & 0.7 & 0.8 & 1.1 & 1.8 \\
\hline GFR, $\mathrm{ml} / \mathrm{min} / 1.73 \mathrm{~m}^{2}$ & & $83^{*}$ & $74^{*}$ & $56^{*}$ & $52^{* *}$ \\
\hline Urinary calcium/creatinine, $\mathrm{mg} / \mathrm{mg}$ & $<0.22$ & 0.56 & ND & ND & 0.05 \\
\hline Urinary magnesium/creatinine, $\mathrm{mg} / \mathrm{mg}$ & $0.154-0.202$ & 0.45 & ND & ND & 0.16 \\
\hline Uric acid, $\mathrm{mg} / \mathrm{dl}$ & $3.4-7.2$ & 6.6 & 7.2 & ND & 11.2 \\
\hline Serum $\mathrm{Na}^{+}, \mathrm{mmol} / \mathrm{l}$ & $136-145$ & 136 & 141 & 142 & 146 \\
\hline Serum $\mathrm{K}^{+}, \mathrm{mmol} / \mathrm{l}$ & $3.5-5.3$ & 3.5 & 3.7 & 4.1 & 4.2 \\
\hline Serum phosphate, mg/dl & $2.4-4.5$ & 6.7 & 5.9 & 5.5 & ND \\
\hline Serum alkaline phosphatase, U/l & $40-129$ & 545 & 387 & 423 & 232 \\
\hline Leukocyturia & & yes & ND & ND & yes \\
\hline Urinary proteins, $\mathrm{g} / 24 \mathrm{~h}$ & $0.04-0.12$ & ND & ND & ND & 0.65 \\
\hline Chronic kidney disease stage & & 2 & 2 & 3 & 3 \\
\hline
\end{tabular}

The GFR was estimated from serum creatinine using the Schwartz formula $\left(^{*}\right)$ and MDRD equation $\left({ }^{* *}\right)$. ND $=$ Not determined.

\begin{abstract}
Multiple sequence alignments of CLDN19 proteins in 18 vertebrate species and other related members of the human claudin superfamily were generated with T-Coffee [11]. The effect of amino acid substitution on protein function was predicted using SIFT (sorting intolerant from tolerant), PolyPhen, PANTHER and Align-GVGD [12-15]. A reference protein sequence (ENSG00000164007) of the human CLDN19 gene was used as the input for SIFT, PolyPhen and PANTHER. Default query options were used for SIFT and PolyPhen prediction. MSA of the CLDN19 proteins in vertebrate species was used as the input MSA for Align-GVGD. The CLDN19 reference protein sequence and mutant CLDN19 sequence were analyzed by the GOR IV secondary structure-prediction method [16].
\end{abstract}

\section{Results}

\section{Clinical Presentation}

The patient with short stature ( -3.2 SDS below the mean for chronological age) was referred for polyuria, polydipsia and recurrent attacks of tetany at the age of 14 years. He was the youngest child in a consanguineous family and was born at term after a normal pregnancy. Laboratory investigations revealed hypomagnesemia, hypocalcemia, hypermagnesuria and hypercalciuria (table 1). Arterial blood gases and serum electrolytes were normal. Radiological examination (fig. $1 b-d$ ) revealed bilateral nephrocalcinosis and renal osteodystrophy including genu valgum (knock-knee) deformity. Ophthal- mologic examination revealed severe ocular abnormalities (fig. 1e-g) including macular colobomata of the left eye and posterior staphyloma in the right eye. The patient had significant myopia with $15^{\circ}$ left exotropia and nystagmoid movement in left gaze. In addition, both eyes showed increased tortuosity of the retinal arteriolar vessels. Since both parents were healthy, the inheritance pattern was likely to be autosomal recessive. The patient was treated with magnesium and calcium salts, calcitriol, potassium citrate and hydrochlorothiazide. Serum calcium and magnesium levels were not normalized after treatment, whereas urinary calcium/creatinine and magnesium/creatinine ratios were reduced.

Screening of the phenotypically unaffected members of the family was performed for hypomagnesemia, hypercalciuria, nephrocalcinosis and nephrolithiasis. One individual (IV-7) revealed bilateral nephrolithiasis (an $8.5-\mathrm{mm}$ stone in the middle calyx of the right kidney and a $12-\mathrm{mm}$ stone at the right UV junction, while an $8.8-\mathrm{mm}$ stone was present in the middle calyx of the left kidney). Two members (IV-1, IV-6) revealed unilateral nephrolithiasis (8.9- and 11-mm stones, respectively) in the middle calyx of the left kidney.

\section{Genotyping and DNA Sequence Analysis}

Genotyping of the patient and 6 normal individuals of the family was performed with microsatellite markers 
mapped in the regions of CLDN16 and CLDN19 genes. The markers were fully informative, and the results revealed that the patient was homozygous for the markers D1S3721, D1S2130 and D1S2645, suggesting linkage to the CLDN19 gene (fig. 1a). However, both the CLDN16 and CLDN19 genes were screened for mutation detection in the proband and his family members. Sequence analysis of exon 3 of the CLDN19 gene revealed a G to A transition at nucleotide position 389 (fig. 1h), resulting in glycine to aspartate (G130D) amino acid substitution. The mutation was confirmed by bidirectional DNA sequencing. The parents (III-1, III-2) and 4 normal individuals (IV-1, IV-4, IV-6 and IV-7) of the family were heterozygous for the mutation. To ensure that the mutation does not represent a neutral polymorphism in the Pakistani population, 100 ethnically matched healthy control individuals were screened for the mutation by PCR followed by direct sequencing and StuI restriction assay (fig. 1i). The mutation was not identified outside the family. Sequence analysis also revealed a synonymous singlenucleotide polymorphism (c.261C>T) in exon 2 of the CLDN19 gene in a homozygous condition in the affected and normal individuals of the family. No other sequence variant was identified in the family in either the CLDN16 or the CLDN19 gene.

\section{Bioinformatic Mutation Analysis}

Multiple alignment of the amino acid sequence of the CLDN19 gene among 18 vertebrate species using T-Coffee revealed $47 \%$ identity and $63 \%$ similarity. The identity and similarity in the third transmembrane domain (TMD) were 17 and 30\%, respectively (among the claudins, the amino acid sequences of the first and fourth transmembrane segments and the first and second extracellular loops are highly conserved, whereas the sequences of the second and third transmembrane domains are more diversified) [17]. The G130 residue in the third TMD was completely conserved among CLDN19 genes across all species (fig. 1j). Multiple sequence alignment of the human CLDN19 with human claudin $1,3,4,7,8,10$, 11 and 16 was also performed. The analysis revealed that G130 was highly conserved (78\%), existing as either a glycine or an alanine (similar hydrophobic) amino acid. These eight claudins, in addition to CLDN19, are known to reside in the distal tubules and collecting ducts, and their roles in paracellular transport of cations in the renal tubules have been demonstrated. The biological importance of these claudin members in renal and other organs has been demonstrated in mutants and gene-targeting studies as well [18]. Evolutionary conservation analysis of mutated amino acid residue G130 utilizing amino acid substitution matrix (PAM 260) revealed a log odd score of 5.0 for a $G$ to $G$ self-substitution, 1.0 for a G to D substitution and $\leq 1.0$ for any other substitution at this position. A lower score indicated that $\mathrm{G}$ to $\mathrm{D}$ is not a favored substitution at this position.

The pathogenicity of G130D mutation was supported by SIFT analysis, which predicted that G130D substitution will affect the protein function with a probability score of 0.00 (the threshold score for intolerance of a substitution was 0.05 ; the amino acids with probabilities of $<0.05$ were predicted to be deleterious). Polyphen analysis predicted G130D substitution to be probably damaging with a score of 2.392 (score ranges from 0 to a positive number, where 0 is neutral, and a high positive number is damaging). PANTHER predicted G130D substitution to have a deleterious effect on protein function. The PANTHER subPSEC (substitution position-specific evolutionary conservation) score was below -3; the previously identified cutoff point for functional significance and the probability of functional impairment $\left(\mathrm{P}_{\text {deleterious }}\right)$ was 0.65 [19]. ALIGN-GVGD analysis predicted that G130D substitution will most likely interfere with protein function (class C65). The GOR IV secondary structure-prediction method predicted that replacement of glycine with aspartic acid at position 130 in the third TMD of claudin-19 may disrupt the arrangement of adjacent extended strands in the protein. This may be due to the fact that glycine is a very unique amino acid in that it contains hydrogen as its side chain and therefore offers extraordinary conformational flexibility.

\section{Discussion}

Epithelia serve as selective and protective barriers in multicellular organisms. Epithelial cells are interconnected by a series of intercellular junctions, collectively known as the epithelial junctional complex, which is composed of tight junctions (TJs), adherens junctions and desmosomes. The TJs are heteromeric protein complexes that form the principal barrier or gate to selectively regulate paracellular diffusion. They also act as a molecular fence to maintain cell polarity by separating the apical and basolateral membrane components. In addition, the TJs act as signaling centers by mediating the bidirectional transmission of information between the environment and the cell interior to control paracellular permeability and differentiation. The major components of the TJs are claudins, which form ion-selective paracel- 
lular pores through the TJs and determine the selectivity of the paracellular transport. Claudins are a large multigene family consisting of 24 members in mammals. The tissue-specific expression of different claudins underlies the different barrier properties seen by different types of epithelia. Claudins bear four transmembrane domains (fig. 1k), a short intracellular NH2-terminal sequence $(\sim 7$ residues), a large first extracellular loop $(\sim 52$ residues) and a shorter second extracellular loop $(\sim 16-33$ residues), and a cytoplasmic $\mathrm{COOH}$-terminal sequence that varies considerably in length ( $\sim 21-63$ residues) between different isoforms $[20,21]$. The only inherited disease known to be caused by claudin mutations so far is FHHNC. The responsible proteins CLDN16 and CLDN19 are required for renal reabsorption of $\mathrm{Mg}^{2+}$ in the TALH [10], where approximately $50-60 \%$ of the filtered $\mathrm{Mg}^{2+}$ is reabsorbed via the paracellular pathway [22]. CLDN16 and CLDN19 completely colocalize in the TALH and thin ascending limbs of Henle. However, CLDN19 is also expressed at high levels in the retina [9]. When coexpressed in a yeast two-hybrid system, CLDN16 and CLDN19 interact in a synergistic manner and form a cation-selective TJ complex [10]. These data explain the fact that CLDN19 mutations are associated with a similar phenotype to that due to CLDN16 mutations except that the patients manifest severe ocular involvement.

In this study, we have identified a novel CLDN19 mutation in a Pakistani patient with FHHNC and severe ocular disease. The identified mutation (G130D) replaces a neutral with a negatively charged amino acid located in the third TMD of claudin-19 protein. Six different mutations have been reported in the CLDN19 gene so far $[9,10$, 23, 24]. The mutations, G20D and P28L lie in the first TMD; V44M and Q57E lie in the first extracellular loop; L90P lies in the second TMD, and G123R lies in the third TMD of the protein. CLDN19 significantly lost its function when any of the mutants (G20D, Q57E, L90P, G123R) were expressed in LLC-PK1 cells. In the yeast two-hybrid assay, G123R mutation severely affected CLDN19 heteromeric interaction with CLDN16 [10]. Moreover, the G130D mutant is homologous to the previously reported claudin-16 mutant G198D, also responsible for FHHNC [25]. Transfection of LLC-PK1 cells with the G198D mutant showed no variation at the level of transcription; while the mutant protein was not detectable with immunofluorescence staining or Western blotting, suggesting that it was instantly degraded [26]. Therefore, the G130D mutation in CLDN19 may be predicted to cause loss of protein function and affect the heteromeric interaction of CLDN19 with CLDN16.
Serum calcium and magnesium levels in the patient presented herein remained low despite administration of calcium and magnesium supplements, as previously reported [3]. Hydrochlorothiazide is known to reduce urinary calcium excretion rates and increase magnesium uptake in a dose-dependent manner [27]. However, in a study of 25 FHHNC families [3], no sustained reduction of hypercalciuria was achieved in the majority of patients with hydrochlorothiazide treatment. In our patient, hydrochlorothiazide administration reduced the urinary calcium/creatinine ratio and magnesium/creatinine ratio (table 1), as previously reported [5]. In contrast, the glomerular filtration rate decreased from $83 \mathrm{ml} / \mathrm{min} /$ $1.73 \mathrm{~m}^{2}$ (stage 2 of chronic kidney disease) at the time of diagnosis to $52 \mathrm{ml} / \mathrm{min} / 1.73 \mathrm{~m}^{2}$ (stage 3 of chronic kidney disease) at the end of follow-up. Therefore, the calcium/creatinine ratio in our patient did not correlate with the extent of renal impairment as previously described $[3,5]$.

The proband presented with severe short stature (-3.2 SDS below the mean for chronological age), and substitution of calcium, calcitriol and hydrochlorothiazide did not improve his height during the follow-up period. Growth failure is a well-documented clinical manifestation in hereditary tubular disorders. However, the median body height in previously reported FHHNC patients varied from -0.9 to -1.7 SDS, while only 1 patient had SDS below $-3.0[5,28]$ and all patients benefited from the substitution of calcium, calcitriol and hydrochlorothiazide [5]. Sanjad et al. [29] recently described extreme short stature (median body height -6.9 SDS) in an FHHNC family with the CLDN16 gene mutation. However, the role of the CLDN16 gene mutation in the short-stature phenotype in their reported family was inconclusive because 1 unaffected sibling, an obligate heterozygote, was also short.

Ultrasongraphic examination of both kidneys of heterozygous members of the affected family revealed either unilateral or bilateral nephrolithiasis in 3 of $6(50 \%)$ individuals. Comparable observations have previously been reported in families affected with FHHNC due to CLDN16 gene mutations [3]. It has been suggested that mutation analysis of families affected by familial hypercalciuria with nephrocalcinosis and/or nephrolithiasis (with an apparently dominant mode of inheritance) would demonstrate heterozygous mutations in the CLDN16 gene in a number of families. A similar relationship may be expected between isolated hypercalciuria/nephrolithiasis and CLDN19 gene mutations. 


\section{Conclusion}

The identification of a novel mutation in the CLDN19 gene strengthens the evidence that FHHNC with severe ocular disease is caused by a dysfunction of the renal TJ protein CLDN19. The study supports the crucial role of CLDN19 in the formation of TJs in the kidneys to maintain magnesium homeostasis and its important role in the eye in the organization and development of the retina. However, further studies are required to establish the exact molecular mechanism of mutations in the development of FHHNC. Understanding the molecular defects of CLDN19 in FHHNC patients should be helpful in the genetic counseling of affected families and may improve specific therapeutic strategies to prevent the development of ESRD in the future.

\section{Acknowledgements}

We wish to thank the family members for their invaluable participation and cooperation. This work was supported by the Higher Education Commission (HEC), Islamabad, Pakistan. Written consent for publication was obtained from the patient and his relatives.

\section{References}

1 Michelis MF, Drash AL, Linarelli LG, De Rubertis ER, Davis BB: Decreased bicarbonate threshold and renal magnesium wasting in a sibship with distal renal tubular acidosis: evaluation of the pathophysiological role of parathyroid hormone. Metabolism 1972;21: 905-920.

-2 Efrati E, Arsentiev-Rozenfeld J, Zelikovic I: The human paracellin-1 gene (hPCLN-1): renal epithelial cell-specific expression and regulation. Am J Physiol Renal Physiol 2005; 288:F272-F283.

3 Weber S, Schneider L, Peters M, Misselwitz J, Ronnefarth G, Boswald M, Bonzel KE, Seeman T, Sulakova T, Kuwertz-Broking E, Gregoric A, Palcoux JB, Tasic V, Manz F, Scharer K, Seyberth HW, Konard M: Novel paracellin-1 mutation in 25 families with familial hypomagnesemia with hypercalciuria and nephrocalcinosis. Am Soc Nephrol 2001;12: 1872-1881.

4 Kuwertz-Broking E, Frund S, Bulla M, Kleta R, August C, Kisters K: Familial hypomagnesemia-hypercalciuria in 2 siblings. Clin Nephrol 2001;56:155-161.

5 Wolf MTF, Dotsch J, Konard M, Boswald M, Rascher M: Follow-up of five patients with FHHNC due to mutations in the Paracellin-1 gene. Pediatr Nephrol 2002;17:602-608.

6 Kari JA, Farouq M, Alshaya HO: Familial hypomagnesemia with hypercalciuria and nephrocalcinosis. Pediatr Nephrol 2003;18: 506-510.

7 Muller D, Kausalya PJ, Bockenhauer D, Thumfart J, Meij IC, Dillon MJ, van't Hoff W, Hunziker W: Unusual clinical presentation and possible rescue of a novel claudin-16 mutation. J Clin Endocrinol Metab 2006;91: 3076-3079.

\section{-}

Weber S, Hoffmann K, Jeck N, Saar K, Boeswald M, Kuwertz-Broeking E, Meij II, Knoers NV, Cochat P, Sulakova T, Bonzel KE, Soergel M, Manz F, Schaerer K, Seyberth HW, Reis A, Konard M: Familial hypomagnesaemia with hypercalciuria and nephrocalcinosis maps to chromosome $3 \mathrm{q} 27$ and is associated with mutations in the PCLN-1 gene. Eur J Hum Genet 2000;8:414-422.

$\checkmark 9$ Konrad M, Schaller A, Seelow D, Pandey AV, Waldegger S, Lesslauer A, Vitzthum H, Suzuki Y, Luk JM, Becker C, Schlingmann KP, Schmid M, Rodriguez-Soriano J, Ariceta G, Cano F, Enriquez R, Juppner H, Bakkaloglu SA, Hediger MA, Gallati S, Neuhauss SCF, Nurnberg P, Weber S: Mutations in the tightjunction gene claudin 19 (CLDN19) are associated with renal magnesium wasting, renal failure, and severe ocular involvement. Am J Hum Genet 2006;79:949-957.

$>10$ Hou J, Renigunta A, Konrad M, Gomes AS, Schneeberger EE, Paul DL, Waldegger S, Goodenough DA: Claudin-16 and claudin-19 interact and form a cation-selective tight junction complex. J Clin Invest 2008; 118:619-628.

11 Notredame C, Higgins DG, Heringa J: TCoffee: a novel method for fast and accurate multiple sequence alignment. J Mol Biol 2000;302:205-217.

12 Ng PC, Henikoff S: Predicting deleterious amino acid substitutions. Genome Res 2001; 11:863-874.

13 Sunyaev S, Ramensky V, Bork P: Towards a structural basis of human non-synonymous single nucleotide polymorphisms. Trends Genet 2000;16:198-199.

14 Thomas PD, Kejariwal A, Campbell MJ, Mi H, Diemer K, Guo N, Ladunga I, UlitskyLazareva B, Muruganujan A, Rabkin S, Vandergriff JA, Doremieux O: PANTHER: a browsable database of gene products organized by biological function, using curated protein family and subfamily classification. Nucleic Acids Res 2003;31:334-341.
15 Tavtigian SV, Deffenbaugh AM,Yin L, Judkins T, Scholl T, Samollow PB, de Silva D, Zharkikh A, Thomas A: Comprehensive statistical study of 452 BRCA1 missense substitutions with classification of eight recurrent substitutions as neutral. J Med Genet 2006; 43:295-305.

16 Garnier J, Gibrat JF, Robson B: GOR secondary structure prediction method. Meth Enzymol 1996;266:540-553.

17 Heiskala M, Peterson PA, Yang Y: The roles of claudin superfamily proteins in paracellular transport. Traffic 2001;2:93-98.

18 Lee NPY, Tong MK, Leung PP, Chan VW, Leung S, Tam PC, Chan KW, Lee KF, Yeung WS, Luk JM: Kidney claudin-19: localization in distal tubules and collecting ducts and dysregulation in polycystic renal disease. FEBS Lett 2006;580:923-931.

-19 Brunham LR, Singaraja RR, Pape TD, Kejariwal A, Thomas PD, Hayden MR: Accurate prediction of the functional significance of single nucleotide polymorphism and mutations in the ABCA1 gene. PLoS Genet 2005; 1:739-747.

20 Terry S, Nie M, Matter K, Balda MS: Rho signaling and tight junction functions. Physiology 2010;25:16-26.

21 Angelow S, Ahlstrom R, Yu ASL: Biology of claudins. Am J Physiol Renal Physiol 2008; 295:867-876.

22 Quamme GA, Dirks JH: Magnesium transport in the nephron. Am J Physiol 1980;239: 393-401.

23 Haisch L, Almeida JR, Abreu da Silva PR, Schlingmann KP, Konrad M: The role of tight junctions in paracellular ion transport in the renal tubule: lessons learned from a rare inherited tubular disorder. Am J Kidney Dis 2011;57:320-330. 
-24 Faguer S, Chauveau D, Cintas P, Tack I, Cointault O, Rostaing L, Vargas-Poussou R, Ribes D: Renal, ocular, and neuromuscular involvements in patients with CLDN19 mutations. Clin J Am Soc Nephrol 2011;6:355360.

-25 Simon DB, Lu Y, Choate KA, Velazquez H, Al-Sabban E, Praga M, Casari G, Bettinelli A, Colussi G, Rodriguez-Soriano J, McCredie D, Milford D, Sanjad S, Lifton RP: Paracellin-1, a renal tight junction protein required for paracellular $\mathrm{Mg}^{2+}$ resorption. Science 1999;285:103-106.

-26 Hou J, Paul DL, Goodenough DA: Parcellin-1 and the modulation of ion selectivity of tight junctions. J Cell Sci 2005;118:51095118 .
7 Frassetto LA, Nash E, Morris RC Jr, Sebastian A: Comparative effects of potassium chloride and bicarbonate on thiazide-induced reduction in urinary calcium excretion. Kidney Int 2000;58:748-752.

28 Haffner D, Weinfurth A, Manz F, Schmidt H, Bremer HJ, Mehls O, Schärer K: Longterm outcome of paediatric patients with hereditary tubular disorders. Nephron 1999; 83:250-260.

29 Sanjad SA, Hariri A, Habbal ZM, Lifton RP: A novel PCLN-1 gene mutation in familial hypomagnesemia with hypercalciuria and atypical phenotype. Pediatr Nephrol 2007; 22:503-508

\section{Web Resources}

Accession numbers and URLs for data presented herein are as follows:

Align GVGD, http://agvgd.iarc.fr/agvgd_input.php

Ensembl genome browser, http://www.ensembl.org

GOR IV secondary structure prediction method, http://npsa-pbil.ibcp.fr/cgi-bin/npsa automat.pl?page $=/ \mathrm{NPSA} /$ npsa_gor4.html

Human gene mutation database, http:// www.hgmd.org

Online mendelian inheritance in man (OMIM), http://www.ncbi.nlm.nih.gov/Omim/

PANTHER classification system, http:// www.pantherdb.org/Tools/csnpScoreForm.jsp

PolyPhen, http://genetics.bwh.harvard.edu/ pph/

SIFT, http://sift.jcvi.org/

T-Coffee multiple sequence alignment program, http://www.ebi.ac.uk/Tools/t-coffee/ index.html

UCSC genome bioinformatics, http:/genome.ucsc.edu 